\title{
Factores que influyen en la elección de una carrera en atención primaria entre los estudiantes de medicina en América Central
}

\author{
Eduardo Benjamín Puertas ${ }^{1}$, Rafael Antonio Orellana², Brian Erazo Muñoz ${ }^{3}$, Jorge Arturo Jiménez ${ }^{4}$, \\ Isamara Gilmiani Medina Quiroz ${ }^{5}$, Lucia Terrón ${ }^{6}$, Alexandre Florencio7, Hilda M. Leal${ }^{8}$, Juan José Vindell²
}

Forma de citar Puertas EB, Orellana RA, Muñoz BE, Jiménez JA, Medina Quiroz IG, Terrón L et al. Factores que influyen en la elección de una carrera en atención primaria entre los estudiantes de medicina en América Central. Rev Panam Salud Publica. 2020;44:e94 https://doi.org/10.26633/RPSP.2020.94

RESUMEN

Objetivos. Identificar las especialidades preferidas, la percepción salarial y los factores que influencian la elección de una carrera en atención primaria en los estudiantes de último año de medicina o en servicio social de América Central.

Métodos. Estudio multicéntrico de corte transversal con aplicación de una encuesta que investigó la información demográfica, la especialidad preferida, la percepción salarial y otros factores que influyen en la elección de la especialidad.

Resultados. Participaron 1722 estudiantes de 31 universidades que identificaron como futuras especialidades: cirugía (11,7\%), ginecología/obstetricia $(10,3 \%)$, pediatría $(9,9 \%)$ y medicina interna $(6,6 \%)$. Medicina general alcanzó 3,8\% y medicina familiar 1,1\%. Al agruparlas el mayor interés fue para las especialidades médicas (49,7\%), seguido de las quirúrgicas (31,7\%). Atención primaria registró un interés del 17,1\%. Las especialidades quirúrgicas fueron percibidas como de mayor ingreso (USD 36 000); atención primaria fue percibida como la de menor ingreso (USD 24 000). El ingreso (23,6\%), el trabajo a futuro (19,7\%) y "hacer una diferencia en las personas" (8,9\%) fueron los principales factores involucrados en la elección. "Trabajar con personas con escaso acceso" se asoció de manera significativa con la preferencia por la atención primaria. La preferencia por otras especialidades fue influenciada por el "prestigio percibido" y "disfrutar la vida" $(P<0,05)$. La mayoría de los participantes que eligieron la atención primaria provenían de una universidad pública $(P<0,05)$, lo que pone de relieve el papel de las instituciones estatales de educación superior.

Conclusiones. Existe una combinación de factores facilitadores y de barreras que inciden en el desinterés hacia las carreras de atención primaria. Se requiere de estrategias desde la academia y el sector gubernamental, así como la definición de políticas públicas, que favorezcan la elección de la atención primaria.

Palabras clave Atención primaria de salud; especialización; fuerza laboral en salud; América Central.

La atención primaria es la provisión de servicios de salud integrados y accesibles por profesionales eficientes en la cobertura de la mayoría de las necesidades de salud de las personas,

\footnotetext{
Organización Panamericana de la Salud, San Salvador, El Salvador. $₫$ Benjamín Puertas, puertasb@paho.org

2 Universidad de El Salvador, San Salvador, El Salvador

3 Universidad Nacional Autónoma de Honduras, Tegucigalpa, Honduras

4 Centro de Desarrollo Estratégico e Información en Salud y Seguridad Social, San José, Costa Rica
}

que desarrollan una relación continuada con sus pacientes, y que realizan su práctica dentro del contexto de la familia y la comunidad (Instituto de Medicina, 1978).

\footnotetext{
5 Universidad Nacional Autónoma de Nicaragua, Managua, Nicaragua

6 Ejercicio Profesional Supervisado Rural, Ciudad de Guatemala, Guatemala.

7 Organización Panamericana de la Salud, Managua, Nicaragua

8 Organización Panamericana de la Salud, San Salvador, El Salvador
} 
Los países con mayor proporción de médicos de atención primaria respecto de su población obtuvieron mejores resultados de salud, incluyendo tasas más bajas de mortalidad general $(1,2)$. En EE.UU. los pacientes que identificaron a los médicos de atención primaria como sus fuentes habituales de atención tuvieron menores tasas de mortalidad en menores de cinco años, en comparación con los pacientes que identificaron a médicos especialistas (3). En Inglaterra, cada médico de cabecera adicional por cada 10000 habitantes se asoció con una disminución del 5\% de la mortalidad (4). La presencia de médicos de atención primaria tuvo un impacto positivo en zonas con alta desigualdad social en México, donde la prestación de atención primaria tuvo un importante efecto sobre la reducción de la mortalidad infantil (5).

Varios países del mundo están experimentando una escasez de personal de salud, en parte debido a la migración y la distribución inequitativa del personal de salud entre los centros urbanos y las zonas rurales. En 2008, en EE.UU. sólo el 37\% de los médicos practicaba atención primaria a pesar de que el $56 \%$ de las visitas a los consultorios médicos eran aptas para este tipo de atención, y un gran porcentaje de la población vivía en áreas con escasez de atención primaria (6). La falta de médicos de familia afecta más a las zonas rurales de los países en desarrollo (7). En los países en desarrollo, las intenciones de carrera de los estudiantes de medicina no están alineadas con las necesidades de los sistemas nacionales de salud, lo que se evidencia en las especialidades que eligen. Los planes de estudio en algunas escuelas de medicina están fuera del contexto de las realidades de las comunidades $(8,9)$.

La escasez de médicos de atención primaria puede explicarse porque los estudiantes de medicina no eligen esta especialidad, los médicos de atención primaria no satisfacen las demandas de una población creciente, y cada vez hay tiempos de espera más largos y menos citas oportunas con el médico (10). Existen varias barreras que explican este fenómeno, como los menores ingresos de la atención primaria en relación con las especialidades (11-19), el bajo prestigio experimentado por las profesiones de atención primaria $(13,18-20)$, y algunos aspectos relacionados con la formación en las escuelas de medicina. En estas, la atención primaria está reflejada de manera insuficiente en los planes de estudio, los estudiantes carecen de exposición a médicos de familia, existe una percepción negativa de los médicos de familia, y prevalece una cultura negativa hacia la atención primaria de la salud $(16,21,22)$.

Sólo un tercio de los estudiantes de medicina de Canadá estaban interesados en ingresar en un programa de medicina familiar (23). La proporción entre médicos generales y especialistas en Chile disminuyó de 8 generalistas por cada 10 especialistas en 1996, a 6 por cada 10 en 2004 (24). En México, la proporción de médicos generales era de $24 \%$, y apenas $8 \%$ eran médicos de familia (25). En Honduras, apenas el $8 \%$ de los médicos que iniciaban el servicio social señaló que seguiría una carrera relacionada con la atención primaria (26), y de ellos apenas el 0,9\% se interesó en la medicina familiar. Los factores que más incidieron en la elección de carrera fueron hacer una diferencia en la vida de las personas $(23,5 \%)$ y el potencial de ingreso económico (13,1\%). Al finalizar el servicio social, el factor que más incidió en los médicos en Honduras fue el potencial de ingreso económico (23,3\%) (27).

El fortalecimiento de programas rurales, el rediseño curricular, el fomento de aplicaciones de estudiantes de sector rural, y las mentorías y tutorías con médicos familiares son alternativas utilizadas por las facultades de medicina de varios países para fomentar la elección de una carrera en atención primaria $(19,21$, 33). La escasez de médicos de atención primaria debe ser cuantificada y proyectada hacia el futuro para conocer su impacto y sus consecuencias y para plantear alternativas de solución, pero la planificación y proyección de las necesidades son circunscritas a la perspectiva de las entidades formadoras y provisoras de servicios de salud. Se necesita más evidencia que permita conocer y actuar sobre los elementos que inciden en la elección de atención primaria.

Este estudio busca identificar las especialidades preferidas, la percepción salarial y los factores que influencian la elección de una carrera en atención primaria en estudiantes de último año de medicina o en servicio social de América Central.

\section{MÉTODOS}

Estudio multicéntrico de corte transversal con estudiantes de último año de medicina o en servicio social de Costa Rica, El Salvador, Guatemala, Honduras y Nicaragua, que investigó cuatro áreas: información demográfica, especialidad preferida, percepción salarial y factores que influyen en la elección de la especialidad. Se seleccionó a estudiantes de último año o en servicio social ya que, por estar al final de la formación, sus intenciones podrían reflejar con mayor certeza la elección final.

La encuesta se adaptó del instrumento elaborado por la Universidad de Alberta, traducido al español para el piloto en

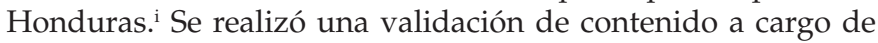
los equipos de investigación de cada país, conformados por académicos, expertos de instituciones participantes, incluyendo asesores de la Organización Panamericana de la Salud (OPS). Los participantes calificaron la importancia percibida de 28 factores que inciden en la elección mediante una escala de Likert. La encuesta se realizó de manera presencial en El Salvador y virtual en los otros cuatro países entre mayo y septiembre de 2019. En El Salvador se realizó en las sedes de las universidades participantes, mientras que en Costa Rica, Guatemala y Honduras se utilizaron Google Forms ${ }^{\circledR}$, y en Nicaragua Question $\mathrm{Pro}^{\circledR}$. Para la percepción salarial, el tipo de cambio a USD según los bancos centrales de los países participantes fue: Honduras (24,34 lempiras, 15 de abril 2019), Nicaragua (32,8489 córdobas, 29 abril), Costa Rica (573,220 colones, 3 de junio), Guatemala (7,4995 quetzales, 10 de septiembre 2019).

El análisis de los datos obtenidos durante el estudio incluyó métodos descriptivos e inferenciales y se utilizó el programa SPSS versión 24 (IBM Corporation). Se calcularon distribuciones de frecuencia y porcentajes; el análisis estadístico incluyó chi cuadrado. Para el análisis multivariado se utilizó el análisis factorial para identificar los factores que tienen una asociación con la elección de la atención primaria, previa la aplicación de la prueba de Kaiser-Meyer-Olkin (KMO) para comprobar si era factible el análisis factorial. Para identificar las diferencias entre determinadas variables se utilizó la prueba no paramétrica de Kruskal Wallis. La significación se estableció en $\mathrm{P}<0,05$.

Las opciones de carrera fueron tomadas del Servicio Canadiense de Pareo de Residentes y adaptadas a las especialidades aprobadas por los colegios médicos de los países participantes. Fueron clasificadas como de atención primaria (medicina

El instrumento empleado está disponible mediante solicitud a los autores 
general, medicina familiar, pediatría y salud pública), medicina de emergencia (medicina de emergencia y cuidados intensivos), especialidad quirúrgica (cirugía general y de especialidad, cirugía plástica, oftalmología, traumatología y ortopedia, otorrinolaringología, urología) o especialidad clínica (anestesiología, cardiología, dermatología, endocrinología, enfermedades infecciosas y tropicales, especialidad pediátrica no quirúrgica, gastroenterología, geriatría, ginecología y obstetricia, inmunología y alergia, medicina física y rehabilitación, medicina interna, nefrología, neumología, neurología, oncología, patología, psiquiatría, radiología e imagen, reumatología).

El protocolo recibió aprobación del Comité de Ética de la OPS/OMS en Washington DC y de los comités de ética de cada país: CEIB-UNAH (Honduras), CENDEISS-CCSS (Costa Rica), Comité Nacional Ética del Ministerio de Salud Pública (Guatemala), CEIB-UNAN León (Nicaragua), CNEIS (El Salvador). Se obtuvo el consentimiento informado por vía física y electrónica y se garantizó el anonimato de los participantes utilizando códigos numéricos en lugar de nombres.

\section{RESULTADOS}

En la encuesta participaron 1722 estudiantes de último año de medicina o en servicio social provenientes de 31 universidades y subsedes de cinco países de América Central: El Salvador (650 participantes), Nicaragua (383), Honduras (308), Guatemala (291) y Costa Rica (90). El número de participantes representó el 57\% del universo de estudiantes de ese nivel en la región. La mayoría $(63,2 \%)$ pertenecía a universidades públicas y una tercera parte $(36,2 \%)$ a universidades privadas. Estaban realizando el servicio socia1 1428 participantes $(82,9 \%)$ y $291(16,9 \%)$ cursaban el último año de la carrera de medicina.

Más de la mitad de los participantes fueron mayores de 25 años (51,7\%); la mayoría fue de sexo femenino (58\%) y se autodefinió como mestizo $(85,7 \%)$. Los participantes provenientes de grupos étnicos y afrodescendientes sumaron 4,7\%; en estos grupos se mantuvo la predominancia del sexo femenino. La mayoría de los estudiantes señalaron que estaban solteros $(92,6 \%)$ y $\sin$ hijos $(90,8 \%)$, aunque $62,9 \%$ pensaba tener hijos a futuro. La mayoría provenía de áreas urbanas (83,3\%), con un $3,1 \%$ de encuestados/as de barrios marginales. Apenas el 1,9\% y el 0,5\% residía en aldeas / caseríos o lugares remotos/aislados, respectivamente. El 91,4\% de participantes respondieron que no tenían progenitores que hubieran ejercido como médico.

\section{Especialidades preferidas}

Las principales especialidades identificadas como futuras carreras fueron: cirugía $(11,7 \%)$, ginecología y obstetricia $(10,3 \%)$, pediatría $(9,9 \%)$, medicina interna $(6,6 \%)$. Dermatología $(4,3 \%)$, cirugía plástica y geriatría $(4,0 \%)$ ocuparon el sexto y séptimo lugar, respectivamente. Medicina general ocupó el octavo lugar $(3,8 \%)$, mientras que salud pública $(1,9 \%)$ y medicina familiar (1,1\%) obtuvieron pocas respuestas (Cuadro 1). Existieron variaciones por país. La segunda opción de especialidad no varió demasiado, aunque las relacionadas con atención primaria obtuvieron respuestas más significativas.

El estudio identificó diferencias en las preferencias de carrera en los países. Las cuatro especialidades básicas se mantuvieron entre los primeros cinco lugares de preferencia, aunque anestesiología se destacó en Costa Rica, Guatemala y El Salvador (tercer, quinto y décimo puesto, respectivamente), y psiquiatría ocupó el cuarto lugar en Costa Rica, séptimo en Nicaragua y octavo en Honduras. Medicina general fue la carrera preferida en Nicaragua $(15,7 \%)$.

Al agrupar las especialidades en categorías se observó más interés en las especialidades médicas $(49,7 \%)$, seguido de las quirúrgicas (31,7\%). Atención primaria alcanzó 17,1\%, aunque eliminando pediatría de este grupo el porcentaje se redujo a $11,7 \%$. Medicina de emergencia fue la preferencia para el 1,6\% de participantes.

CUADRO 1. Comparación de las especialidades preferidas por subregión, país y clasificación

\begin{tabular}{|c|c|c|c|c|c|c|c|c|c|c|c|c|}
\hline \multirow[t]{2}{*}{ Especialidades } & \multicolumn{2}{|c|}{ América Central } & \multicolumn{2}{|c|}{ Costa Rica } & \multicolumn{2}{|c|}{ El Salvador } & \multicolumn{2}{|c|}{ Guatemala } & \multicolumn{2}{|c|}{ Honduras } & \multicolumn{2}{|c|}{ Nicaragua } \\
\hline & $\mathrm{n}(\%)$ & Clasif & $\mathrm{n}(\%)$ & Clasif & $\mathrm{n}(\%)$ & Clasif & $\mathrm{n}(\%)$ & Clasif & $\mathrm{n}(\%)$ & Clasif & $n(\%)$ & Clasif \\
\hline Cirugía & $199(11,7)$ & 1 & $5(6,0)$ & $4^{b}$ & $96(15,0)$ & 1 & $21(7,2)$ & $4^{b}$ & $29(9,4)$ & $3^{b}$ & $48(12,5)$ & $3^{b}$ \\
\hline Ginecología & $176(10,3)$ & 2 & $8(9,5)$ & $1^{\mathrm{a}}$ & $73(11,4)$ & $3^{b}$ & $63(21,6)$ & $1^{\mathrm{a}}$ & $31(10,1)$ & 2 & $1(0,3)$ & $17^{b}$ \\
\hline Pediatría & $168(9,9)$ & 3 & $3(3,6)$ & $5^{b}$ & $81(12,7)$ & $2^{a}$ & $40(13,7)$ & $2^{a}$ & $40(13,0)$ & $1^{\mathrm{a}}$ & $4(1,0)$ & $14^{\mathrm{b}}$ \\
\hline Medicina interna & $113(6,6)$ & 4 & $6(7,1)$ & $2^{\mathrm{a}}$ & $65(10,2)$ & 4 & $13(4,5)$ & $6^{b}$ & $23(7,5)$ & 4 & $6(1,6)$ & $12^{b}$ \\
\hline Traumatología & $84(4,9)$ & 5 & $3(3,6)$ & 5 & $30(4,7)$ & $7^{b}$ & $32(11,0)$ & $3^{\mathrm{a}}$ & $16(5,2)$ & $6^{b}$ & $4(1,0)$ & $14^{b}$ \\
\hline Dermatología & $73(4,3)$ & 6 & $4(4,8)$ & $4^{\mathrm{a}}$ & $28(4,4)$ & $8^{b}$ & $12(4,1)$ & $7^{b}$ & $8(2,6)$ & $11^{b}$ & $21(5,5)$ & $5^{\mathrm{a}}$ \\
\hline Cirugía plástica & $68(4,0)$ & 7 & $3(3,6)$ & $5^{\mathrm{a}}$ & $31(4,9)$ & $5^{\mathrm{a}}$ & $6(2,1)$ & $11^{\mathrm{b}}$ & $17(5,5)$ & $5^{a}$ & $11(2,9)$ & $8^{b}$ \\
\hline Geriatría & $68(4,0)$ & 7 & $4(4,8)$ & $4^{\mathrm{a}}$ & $8(1,3)$ & $5^{b}$ & $2(0,7)$ & $15^{b}$ & $1(0,3)$ & $18^{b}$ & $53(13,8)$ & $2^{\mathrm{a}}$ \\
\hline Patología & $58(3,4)$ & 9 & $1(1,2)$ & $7^{\mathrm{a}}$ & $11(1,7)$ & $13^{b}$ & $2(0,7)$ & $15^{b}$ & $2(0,6)$ & $17^{b}$ & $42(11,0)$ & $4^{\mathrm{a}}$ \\
\hline Anestesiología & $51(3,0)$ & 10 & $5(6,0)$ & $3^{\mathrm{a}}$ & $18(2,8)$ & 10 & $15(5,2)$ & $5^{\mathrm{a}}$ & $6(1,9)$ & $13^{b}$ & $7(1,8)$ & $11^{\mathrm{b}}$ \\
\hline Psiquiatría & $47(2,8)$ & 11 & $4(4,8)$ & $4^{\mathrm{a}}$ & $11(1,7)$ & $13^{b}$ & $4(1,4)$ & $13^{b}$ & $14(4,5)$ & $8^{a}$ & $14(3,7)$ & $7^{\mathrm{a}}$ \\
\hline \multicolumn{13}{|l|}{ Atención primaria } \\
\hline Medicina general & $64(3,8)$ & 8 & $0(0,0)$ & $35^{b}$ & $2(0,3)$ & $19^{b}$ & $0(0,0)$ & $35^{b}$ & $2(0,6)$ & $17^{b}$ & $60(15,7)$ & $1^{\mathrm{a}}$ \\
\hline Salud pública & $32(1,9)$ & 18 & $0(0,0)$ & $35^{b}$ & $15(2,4)$ & $12^{\mathrm{a}}$ & $1(0,3)$ & $16^{\mathrm{a}}$ & $2(0,6)$ & $17^{a}$ & $14(3,7)$ & $7^{\mathrm{a}}$ \\
\hline Medicina familiar & $19(1,1)$ & 23 & $3(3,6)$ & $5^{a}$ & $8(1,3)$ & $14^{\mathrm{a}}$ & $3(1,0)$ & $14^{\mathrm{a}}$ & $5(1,6)$ & $14^{\mathrm{a}}$ & $0(0,0)$ & $35^{\mathrm{b}}$ \\
\hline
\end{tabular}


CUADRO 2. Características de los participantes por categoría de especialización preferida

\begin{tabular}{|c|c|c|c|c|c|}
\hline \multicolumn{6}{|c|}{ Especialidades categorizadas } \\
\hline Demográficas & $\begin{array}{c}\text { Atención } \\
\text { primaria } \\
\mathrm{N}(\%) \\
(\mathrm{n}=283)\end{array}$ & $\begin{array}{c}\text { Especialidad } \\
\text { quirúrgica } \\
N(\%) \\
(n=525)\end{array}$ & $\begin{array}{c}\text { Medicina } \\
\text { emergencia } \\
\mathrm{N}(\%) \\
(\mathrm{n}=26)\end{array}$ & $\begin{array}{c}\text { Especialidad } \\
\text { médica } \\
\mathrm{N}(\%) \\
(\mathrm{n}=824)\end{array}$ & $\begin{array}{c}\text { Total } \\
N(\%) \\
(n=1658)\end{array}$ \\
\hline \multicolumn{6}{|l|}{ Año cursadoa } \\
\hline Último año & $42(14,6)$ & $78(27,2)$ & $10(3,5)$ & $157(54,7)$ & $287(100,0)$ \\
\hline Año social & $241(17,6)$ & $446(32,6)$ & $16(1,2)$ & $665(48,6)$ & $1383(100,0)$ \\
\hline \multicolumn{6}{|l|}{ Edad } \\
\hline$<25$ años & $144(17,8)$ & $266(32,8)$ & $14(1,7)$ & $386(47,7)$ & $810(100,0)$ \\
\hline$>25$ años & $139(16,4)$ & $259(30,5)$ & $12(1,4)$ & $438(51,7)$ & $848(100,0)$ \\
\hline Total & $283(17,1)$ & $525(31,7)$ & $26(1,6)$ & $824(49,7)$ & $1658(100,0)$ \\
\hline \multicolumn{6}{|l|}{ Sexo } \\
\hline Blanco & $26(16,6)$ & $50(31,8)$ & $2(1,3)$ & $79(50,3)$ & $157(100,0)$ \\
\hline Mestizo & $236(16,7)$ & $461(32,5)$ & $22(1,6)$ & $698(49,3)$ & $1417(100,0)$ \\
\hline Afrodescendiente & $7(33,3)$ & $0(0,0)$ & $0(0,0)$ & $14(66,7)$ & $21(100,0)$ \\
\hline Grupo étnico & $14(23,3)$ & $12(20,0)$ & $2(3,3)$ & $32(53,3)$ & $60(100,0)$ \\
\hline Otro & $0(0,0)$ & $2(66,7)$ & $0(0,0)$ & $1(33,3)$ & $3(100,0)$ \\
\hline Total & $283(17,1)$ & $525(31,7)$ & $26(1,6)$ & $824(49,7)$ & $1658(100,0)$ \\
\hline \multicolumn{6}{|l|}{ Estado civil } \\
\hline Soltero(a) & $258(16,8)$ & $496(32,2)$ & $24(1,6)$ & $762(49,5)$ & $1540(100,0)$ \\
\hline Casado/Unión libre & $25(21,9)$ & $28(24,6)$ & $2(1,8)$ & $59(51,8)$ & $114(100,0)$ \\
\hline Separado(a)/Viudo(a) & $0(0,0)$ & $1(25,0)$ & $0(0,0)$ & $3(75,0)$ & $4(100,0)$ \\
\hline Total & $283(17,1)$ & $525(31,7)$ & $26(1,6)$ & $824(49,7)$ & $1658(100,0)$ \\
\hline No & $261(17,2)$ & $467(30,8)$ & $25(1,7)$ & $761(50,3)$ & $1514(100,0)$ \\
\hline Total & $283(17,1)$ & $525(31,7)$ & $26(1,6)$ & $824(49,7)$ & $1658(100,0)$ \\
\hline
\end{tabular}

Estadísticamente significativo $(P<0,05)$

Fuente: Elaborado por los autores a partir de los datos del estudio

Las especialidades médicas ( $56,2 \%$ vs. $40,4 \%$ ) y de atención primaria $(18,5 \%$ vs. $15,1 \%)$ prevalecieron en la población de sexo femenino. Las especialidades quirúrgicas $(42,5 \%$ vs. $24,1 \%)$ y de medicina de emergencia ( $2 \%$ vs. $1,2 \%$ ) prevalecieron entre los hombres.

De las características sociodemográficas analizadas, resultaron estadísticamente significativas el año cursado y el sexo $(\mathrm{P}<0,05)$, con respecto a las categorías de especialidades. Las especialidades médicas fueron las preferidas por los estudiantes de último año y en servicio social $(54,7 \%$ y $48,6 \%)$, especialmente de sexo femenino (56,2\%). (Cuadro 2 )

El 71\% de los participantes que eligieron la opción de atención primaria provenían de una universidad pública $(\mathrm{P}<0,05)$. El 49,6\% de la población encuestada consideró que su elección no era definitiva, comparado con el 39,1\% que sí estaba seguro de su decisión.

\section{Percepción salarial}

La mediana de ingreso anual que esperaban ganar los participantes cinco años después de culminada la carrera fue de USD 30 000. Cerca del $65 \%$ de los encuestados creía que el salario de los médicos generales era inferior a USD 1500 luego de 2-3 años de haber concluido la carrera, en comparación con el $59 \%$ en el caso de médicos de familia y $29 \%$ en el caso de pediatría. El 19\% que eligió otras especialidades aspiraba a un salario entre USD 3000 y 4 000, mientras que el 11,6\% aspiraba ganar USD 4000 o más.

El $36,7 \%$ de las mujeres consideraron que el salario de un médico general era inferior a USD 1500 en comparación al $27,6 \%$ de los hombres. Similares diferencias se identificaron con relación a medicina familiar ( $33,4 \%$ vs. $24,8 \%$ ).

Las especialidades quirúrgicas tuvieron la percepción de mayor ingreso, con una mediana de USD 36 000, mientras que 
FIGURA 1. Percepción salarial por categoría de especialidad y país, en dólares estadounidenses

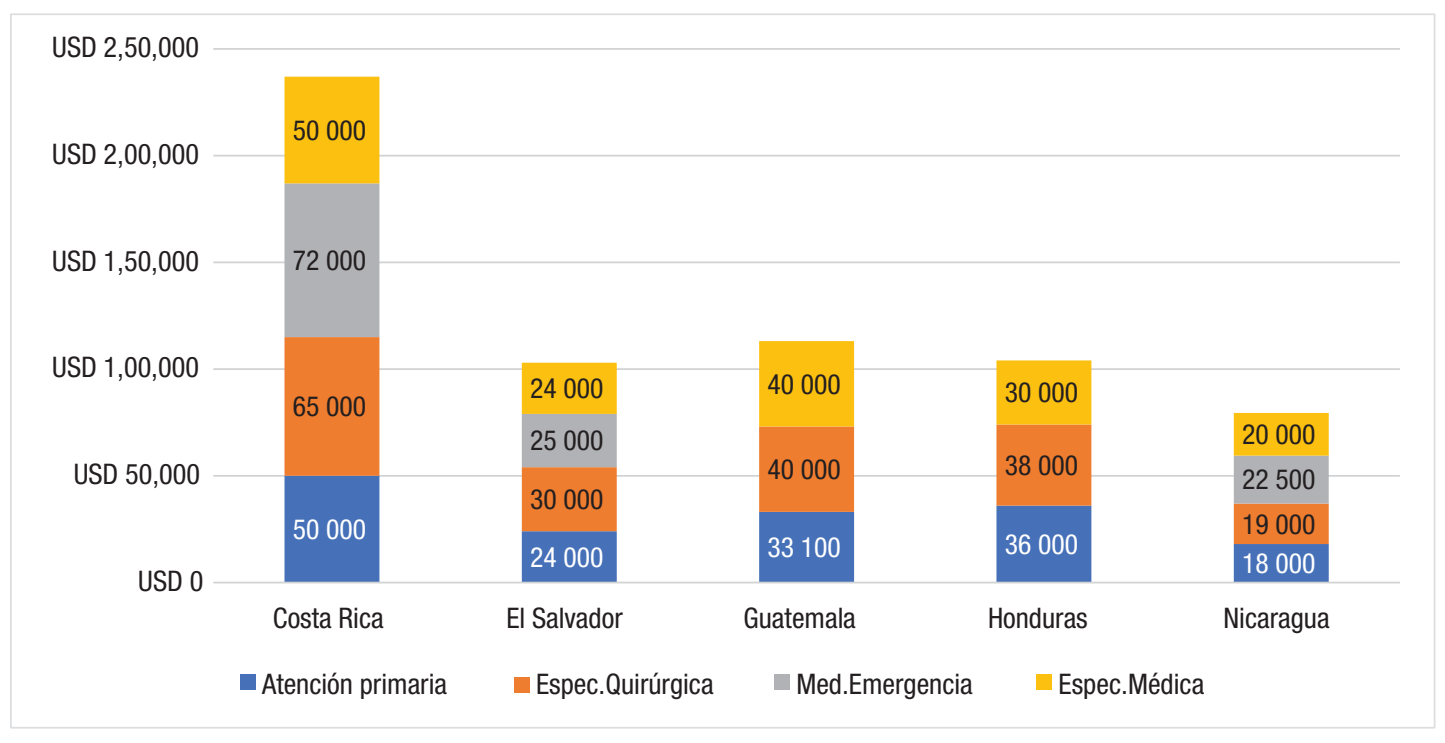

Fuente: Elaborado por los autores a partir de los datos del estudio

USD, dólares estadounidenses (tipo de cambio al 15 de mayo de 2019 según la tabla de Naciones Unidas UN Operational Rates of Exchange)

las carreras de atención primaria tuvieron la percepción más baja con una mediana de USD 24 000, es decir 50\% más baja que las quirúrgicas. Cuando se agrupó las carreras en "atención primaria" y "otras especialidades", se identificó una diferencia estadísticamente significativa $(\mathrm{P}<0,05)$ en la percepción salarial anual.

La percepción salarial más alta se observó en Costa Rica y la más baja en Nicaragua. Las carreras de atención primaria tuvieron una percepción baja en general, aunque en Honduras la mediana fue mayor a la de las especialidades médicas. (Figura 1)

\section{Factores influyentes en la elección de carrera}

Los participantes consideraron como primer factor más importante: ingreso potencial $(23,6 \%)$, potencial de trabajo a futuro $(19,7 \%)$, "hacer una diferencia positiva en las personas" $(8,9 \%)$, prestigio percibido $(8,9 \%)$ y oportunidad por enseñar $(8,1 \%)$ (Figura 2). "Potencial de trabajo a futuro" fue seleccionado como el segundo y tercer factor más importante que influyó en la elección de los participantes.

Al analizar por países, los factores más influyentes se mantuvieron, solo cambiando el primer lugar de preferencia. El ingreso potencial fue el factor más importante para los participantes de Guatemala (43\%) y El Salvador (24,7\%), mientras que para Costa Rica $(30,2 \%)$ y Nicaragua $(20,1 \%)$ fue el potencial de trabajo. En el caso de Honduras, el prestigio percibido de la especialidad $(23,7 \%)$ fue el factor más importante.

$\mathrm{El}$ factor "oportunidad de trabajar con personas con escaso acceso a la atención" fue significativamente asociado con la preferencia por atención primaria, comparado con otras especialidades combinadas (77,5\% vs 73,0\%, P < 0,05). En contraste, los estudiantes que prefirieron otras especialidades fueron más influenciados por el prestigio percibido $(68,6 \%$ vs $60,9 \%$, $\mathrm{P}<0,05)$ y por la "oportunidad de disfrutar la vida fuera del trabajo" (76,2\% vs $68,7 \%)$.
Cuando todas las especialidades se combinaron, las variables "preferencia por trabajar en una comunidad rural"; "influencia familia, amigos o comunidad"; "oportunidad de trabajar con problemas médicos agudos"; "duración de la residencia; habilidad para utilizar un amplio rango de técnicas y conocimientos en el cuidado del paciente"; "habilidad de dominar un grupo pequeño de técnicas"; "oportunidades para trabajar con independencia profesional"; "desarrollo de relaciones largas con pacientes"; y "oportunidad de disfrutar la vida fuera del trabajo" resultaron estadísticamente significativas $(\mathrm{P}<0,05)$. (Cuadro 3)

La prueba de KMO dio un valor de 0,958, lo que permitió proceder con el análisis factorial. Este identificó cuatro categorías de factores correlacionados, cuyos componentes resultantes se agruparon en: (a) factores institucionales, (b) personales, (c) ingreso y reconocimiento profesional, $y$, (d) factores comunitarios que influencian la elección de carrera en atención primaria. El nivel de significancia de estas variables estuvo por encima de 0,05 , por lo que no se continuó con el modelo de regresión logística.

La población participante en el estudio identificó otros factores que incidieron en su elección de carrera. Las 197 opciones de factores fueron agrupadas en las siguientes categorías: ambiente laboral, desarrollo profesional, armonía de vida laboral y personal, accesibilidad. El 25,8\% estuvo relacionado con el ambiente laboral, seguido de armonía de la vida laboral y personal $(12,2 \%)$.

\section{DISCUSIÓN}

El grupo participante fue representativo de la población centroamericana en términos de composición demográfica. La mayoría era de origen mestizo y la población afrodescendiente y de otras etnias fue mínima, lo que demuestra el déficit en la formación de médicos provenientes de estos grupos prioritarios. Los participantes provenientes de zonas rurales o 
FIGURA 2. Carrera en atención primaria: facilitadores, barreras y factores más influyentes

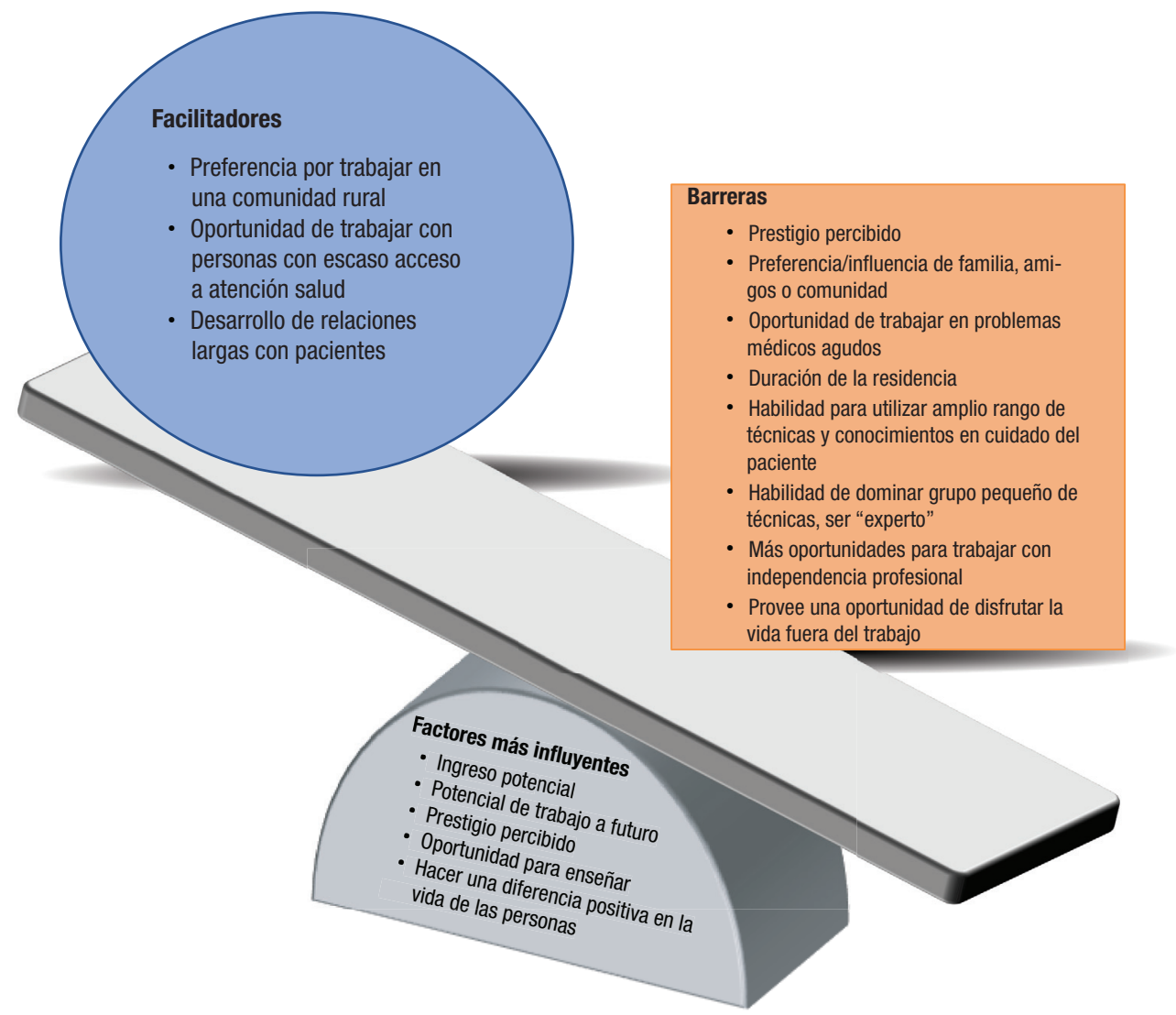

Fuente: Elaborado por los autores a partir de los datos del estudio

remotas fueron muy pocos; esto tiene un impacto negativo en la distribución de personal, dado que la evidencia señala que es más factible que los profesionales de salud que provienen de zonas rurales ejerzan en lugares alejados de los centros urbanos $(28,29)$. La escasa presencia de participantes de barrios marginales tiene implicaciones similares, y puede estar relacionada con las pocas oportunidades de desarrollo, el bajo nivel de escolaridad y los altos niveles de inseguridad en esas zonas, aunque están más cerca de instituciones de educación superior.

Más de la mitad de los participantes fueron mayores de 25 años y de sexo femenino. La feminización de la carrera médica fue evidente y debe tomarse en cuenta durante la planificación de recursos humanos para la salud. La mayoría de participantes estaba soltera y sin hijos, aunque el $63 \%$ pensaba tenerlos a futuro. Con excepción de la edad, las características demográficas no fueron indicativas de una elección de carreras de atención primaria, aunque la evidencia señala que los estudiantes casados o unidos, mayores de 25 y con niños tenían más interés en escoger atención primaria como carrera (13,30-34).

Las especialidades preferidas fueron cirugía, ginecología/ obstetricia, pediatría y medicina interna, resultados consistentes con los de otros estudios (8,20,24,35-37). Medicina familiar fue de las especialidades menos mencionadas. Llama la atención que dermatología y cirugía plástica se encuentren dentro de las diez principales especialidades, cuando el perfil epidemiológico de la población centroamericana tiene problemas más relacionados con enfermedades transmisibles, no transmisibles y problemas sociales como violencia y accidentes de tránsito. Medicina general ocupó el octavo lugar debido a que los participantes de Nicaragua la ubicaron como primera elección, aunque en los otros países no aparece entre las diez primeras (en Costa Rica fue la última en la clasificación). Las carreras de atención primaria obtuvieron respuestas más significativas como segunda opción, lo que significa que existe un grupo de participantes que no ha descartado la opción de atención primaria como elección.

La ausencia de guardias nocturnas y de llamadas de emergencia pueden haber incidido en la preferencia por dermatología, especialmente en participantes de sexo femenino que consideran formar una familia y tener hijos $(13,34,35)$.

Las enfermedades infecciosas y tropicales apenas fueron mencionadas como preferencia de carrera, aunque América Central tiene alta incidencia de dengue, chikungunya y zika. Es de destacar el poco interés en medicina de emergencia en una región con altas tasas de accidentes de vehículos a motor y con índices de violencia que sobrepasan los 50 homicidios por 100000 personas (OMS, 2014). De los países del Triángulo Norte, solo en Honduras la psiquiatría se ubicó dentro de las diez especialidades preferidas. Fue interesante comprobar que la geriatría apareció en la lista principal a diferencia de lo observado en estudios similares en Honduras $(26,27)$, lo que se corresponde con la transición demográfica y el crecimiento de la población adulta mayor en la región.

Cuando se agrupó a las especialidades y carreras en categorías, las especialidades médicas fueron las de mayor interés, 


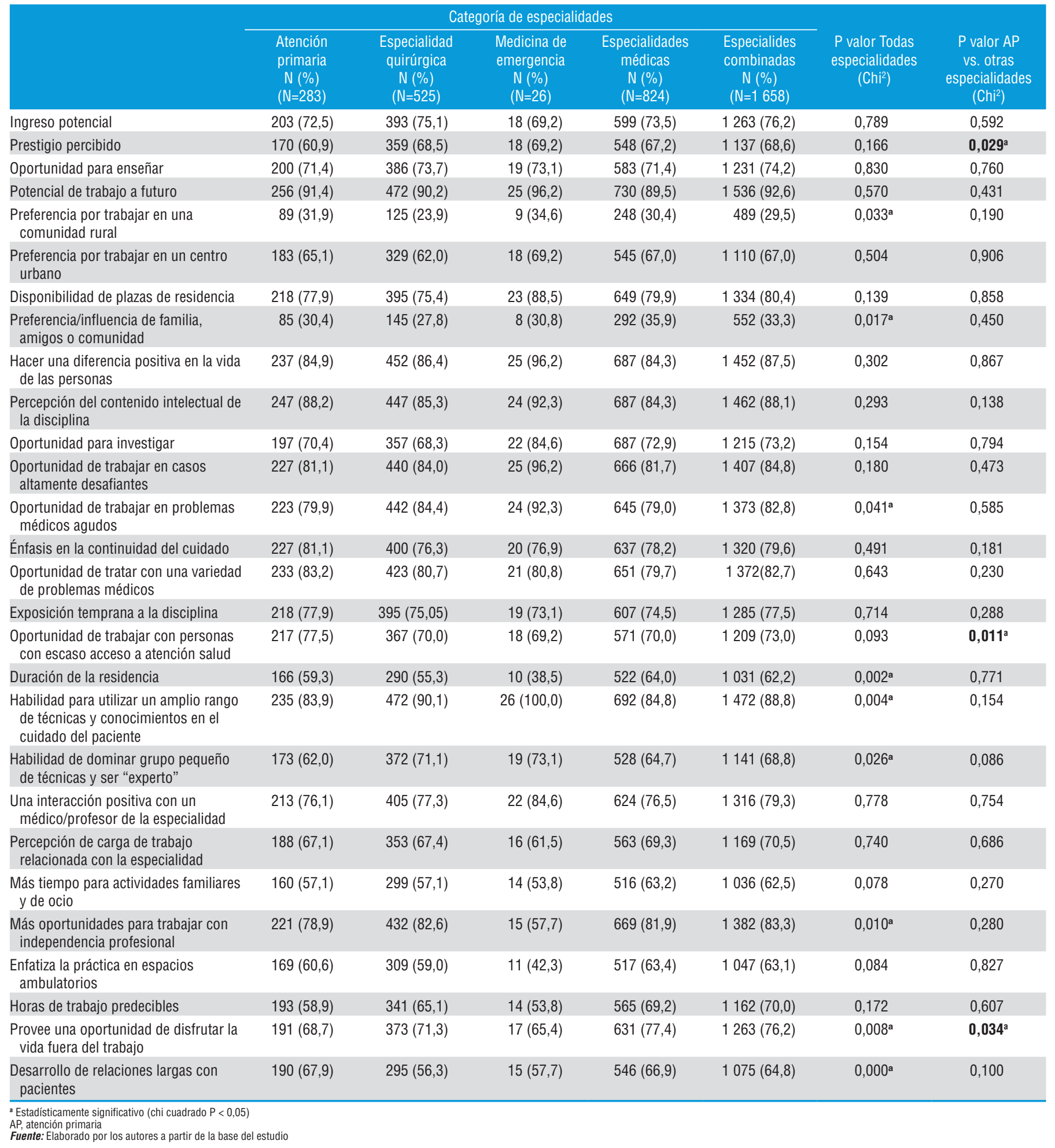

seguidas de las quirúrgicas y atención primaria. Al desagregar pediatría de la categoría, el interés por la atención primaria se redujo a $11,4 \%$, un dato más comparable con estudios similares $(26,27)$. El interés por la atención primaria en América Central fue menor que el reportado en estudios efectuados en Canadá, Chile, Alemania, Francia y Estados Unidos (24,30,35-37), lo que genera dudas sobre la operacionalización del modelo de salud basado en la atención primaria, que los países participantes destacan como parte del sistema de salud.

La indecisión en $11 \%$ de participantes y el hecho de que sea una probabilidad en la mitad de ellos deja espacio para motivar a los estudiantes de último año o en servicio social a que aspiren 
por carreras de atención primaria. El servicio social podría ser el entorno propicio para que el trabajo comunitario y las acciones de promoción, prevención y participación social generen interés en la atención primaria. Sin embargo, el servicio social puede ser desmotivante debido al abandono que sufren los estudiantes, la falta de acceso a insumos y equipos, las pocas opciones de desarrollo profesional y, en muchos casos, la inseguridad.

Las mujeres prefirieron las especialidades médicas y de atención primaria, mientras que en los hombres prevalecieron las especialidades quirúrgicas y de medicina de emergencia. Los resultados fueron significativos en cuanto a la diferencia entre la categoría de especialidades y el sexo de los participantes. La diferencia más marcada entre hombres y mujeres se observó en las especialidades quirúrgicas, que posiblemente siguen siendo un bastión masculino pues requieren muchas horas de dedicación hospitalaria y guardias nocturnas. Los estudios refieren que en el campo de la medicina las mujeres están más interesadas en tener menos horas de trabajo o mayor flexibilidad en comparación con sus colegas masculinos o de menor edad $(39,40)$. Los resultados son comparables a la evidencia encontrada, donde hubo predominancia de mujeres en las carreras de atención primaria como elección preferida $(17,20,23,40)$.

La mayoría de los participantes que eligieron la atención primaria provenían de una universidad pública. Esta diferencia fue significativa y pone de relieve el papel de las instituciones de educación superior estatales como formadoras de recursos humanos con un enfoque integral y con una visión de responsabilidad social.

La percepción salarial de la población encuestada fue menor para la categoría de atención primaria en comparación con las otras especialidades. La percepción más alta se observó en las especialidades quirúrgicas, cuya mediana fue $50 \%$ mayor que la de atención primaria. Estos datos son consistentes con los factores identificados que inciden en la elección, específicamente con el potencial de ingreso. La evidencia corrobora estos resultados $(11,13,15)$. En Honduras no existe una diferencia marcada entre los salarios de especialistas y médicos generales, que están regulados por la Ley del Estatuto del Médico Empleado, lo que podría explicar que la percepción salarial para la atención primaria fue más alta que para las especialidades clínicas.

Los participantes consideraron como primer factor más importante al "ingreso potencial", el "potencial de trabajo", "hacer una diferencia positiva en las personas" o el "prestigio percibido". El potencial de trabajo fue seleccionado como el segundo y tercer factor más importante y para Costa Rica fue el factor más decisivo, lo que conduce a considerar que podría estar prevaleciendo aun por encima de las preferencias personales. El factor "oportunidad de trabajar con personas con escaso acceso a la atención en salud" estuvo relacionado con la elección de atención primaria, mientras que los factores "prestigio percibido" y "oportunidad de disfrutar la vida" influyeron en la elección de otras especialidades. Cabe destacar que "disfrutar la vida fuera del trabajo" es un factor que incidió en los estudiantes de medicina al combinar todas las especialidades o al analizarlas en función de la atención primaria. La mayoría de los factores que incidieron en la decisión de carrera en la población participante representaron barreras para la elección de atención primaria (Figura 2) y fueron similares a aquellos identificados en la literatura $(14,19,30-32,34)$ y en estudios previos realizados en Honduras $(26,27)$.

El estudio presenta algunas limitaciones. Incluyó a estudiantes de último año de medicina o en servicio social, por lo que los resultados no son generalizables a todos los estudiantes. Podría haber alguna diferencia de percepción sobre la elección de carrera entre estudiantes de último año y los de servicio social, pero dado que el tiempo que separa ambos periodos es escaso la diferencia sería mínima. El cuestionario midió la elección "preferida" de los alumnos de la carrera de medicina, y no la elección "real".

En conclusión, existe una combinación de factores que inciden en la elección de carrera. Aunque algunos fueron considerados facilitadores en la elección de atención primaria, la mayoría fueron barreras, lo que explica el bajo interés de los participantes. Los resultados del estudio pueden servir para incidir en la definición de políticas públicas y estrategias que apoyen la atención primaria, que desde la academia se enfoquen en la admisión, formación y mentoría de estudiantes de medicina, y desde el sector gubernamental en el otorgamiento de becas de especialidad en carreras de atención primaria, la planificación de recursos humanos para la salud y la contratación de personal de atención primaria en las entidades provisoras de salud en América Central.

Contribuciones de los autores. EBP concibió y planificó el estudio original; RAO, BEM, JAJ, IGMQ, LT, HML y AF planificaron y recolectaron los datos de país. EBP, RAO, BEM y JJV analizaron los datos e interpretaron los resultados. EBP y JJV escribieron el manuscrito. Todos los autores revisaron el manuscrito y aprobaron la versión final.

Agradecimientos. A los equipos nacionales que trabajaron en la preparación y realización de la encuesta, la digitalización de los datos y la consolidación de la base de datos en los cinco países; a la Organización Panamericana de la Salud OPS/OMS por su apoyo técnico y logístico; y a los participantes provenientes de las facultades de medicina de América Central.

\section{Conflicto de intereses. Ninguno declarado.}

Declaración. Las opiniones expresadas en este manuscrito son únicamente responsabilidad de los autores y no reflejan necesariamente los criterios ni la política de la $R P S P / P A J P H$ o la Organización Panamericana de la Salud.

\section{REFERENCIAS}

1. Starfield B, Shi L, Macinko J. Contribution of primary care to health systems and health. Milbank Q. 2005;83(3):457-502. doi: 10.1111/j.1468-0009.2005.00409.x.

2. Villalbi JR, Guarga A, Pasarin MI, et al. An evaluation of the impact of primary care reform on health. Aten Primaria. 1999;24(8): 468-74.
3. Friedberg MW, Hussey PS, Schneider EC. Primary care: A critical review of the evidence on quality and costs of health care. Health Aff (Millwood). 2010;29(5):766-772. doi: 10.1377/hlthaff.2010.0025

4. Gulliford MC. Availability of primary care doctors and population health in England: Is there an association? J Public Health Med. 2002;24(4):252-54. 
5. Reyes H, Perez-Cuevas R, Salmeron J, Tome P, Guiscafre H, Gutierrez G. Infant mortality due to acute respiratory infections: The influence of primary care processes. Health Policy Plan. 1997;12(3):214-23.

6. Bodenheimer T, Pham HH. Primary care: Current problems and proposed solutions. Health Aff (Millwood). 2010;29(5):799-805. doi: $10.1377 /$ hlthaff.2010.0026

7. Lehmann U, Dieleman M, Martineau T. Staffing remote rural areas in middle- and low-income countries: A literature review of attraction and retention. BMC Health Serv Res. 2008;8:19-6963-8-19. doi: 10.1186/1472-6963-8-19

8. Burch VC, McKinley D, van Wyk J, et al. Career intentions of medical students trained in six Sub-saharan African countries. Educ Health (Abingdon). 2011;24(3):614.

9. Greysen SR, Dovlo D, Olapade-Olaopa EO, Jacobs M, Sewankambo N, Mullan F. Medical education in Sub-saharan Africa: A literature review. Med Educ. 2011;45(10):973-986. doi: 10.1111/j.1365-2923. 2011.04039.x

10. Bodenheimer T. Primary care: will it survive? N Engl J Med. 2006;355(9):861-4.

11. Grayson MS, Newton DA, Thompson LF. Payback time: The associations of debt and income with medical student career choice. Med Educ. 2012;46(10):983-991. doi: 10.1111/j.1365-2923.2012. 04340.x

12. Phillips JP, Wilbanks DM, Rodriguez-Salinas DF, Doberneck DM. Specialty income and career decision making: a qualitative study of medical student perceptions. Medical Education. 2019;53 (6):593-604

13. Newton DA, Grayson MS, Thompson LF. Money, lifestyle, or values? why medical students choose subspecialty versus general pediatric careers. Clin Pediatr (Phila). 2010;49(2):116-22. doi: 10.1177/ 0009922809350216

14. Teitelbaum HS, Ehrlich N, Travis L. Factors affecting specialty choice among osteopathic medical students. Acad Med. 2009;84(6):718-23. doi: 10.1097/ACM.0b013e3181a43c60

15. Morra DJ, Regehr G, Ginsburg S. Medical students, money, and career selection: Students' perception of financial factors and remuneration in family medicine. Fam Med. 2009;41(2):105-10

16. Bazargan M, Lindstrom RW, Dakak A, Ani C, Wolf KE, Edelstein RA. Impact of desire to work in underserved communities on selection of specialty among fourth-year medical students. J Natl Med Assoc. 2006;98(9):1460-65.

17. Rosenblatt RA, Andrilla CH. The impact of U.S. medical students' debt on their choice of primary care careers: An analysis of data from the 2002 medical school graduation questionnaire. Acad Med. 2005;80(9):815-9.

18. Tolhurst H, Stewart M. Becoming a GP: a qualitative study of the career interests of medical students. Aust Fam Physician. 2005;34(3):204-6.

19. Senf JH, Campos-Outcalt D, Kutob R. Factors related to the choice of family medicine: A reassessment and literature review. J Am Board Fam Pract. 2003;16(6):502-12.

20. Compton MT, Frank E, Elon L, Carrera J. Changes in U.S. medical students' specialty interests over the course of medical school. J Gen Intern Med. 2008;23(7):1095-100. doi: 10.1007/s11606-008-0579-z

21. Scott I, Wright B, Brenneis F, Brett-Maclean P, McCaffrey L. Why would I choose a career in family medicine? Reflections of medical students at 3 universities. Can Fam Physician. 2007;53 (11):1956-7

22. Erikson CE, Danish S, Jones KC, Sandberg SF, Carle AC. The role of medical school culture in primary care career choice. Acad Med. 2013;88(12):1919-26. doi: 10.1097/ACM.0000000000000038

23. Vanasse A. Attractiveness of family medicine for medical students influence of research and debt. Can Family Physician. 2011;57(6):e216.
24. Breinbauer KH, Fromm RG, Fleck LD, Araya CL. Trends among medical students towards general practice or specialization. Rev Med Chil. 2009;137(7):865-72. doi: /S0034-98872009000700001

25. Santacruz Varela J, Fajardo Dolci G, Hernández Torres F, Real Tovar FA. Metodología para estimar el número de médicos especialistas en México. En: Fajardo Dolci G, Santacruz Varela J, Lavalle Montalvo C, eds. La formación de médicos especialistas en México. Ciudad de México: Intersistemas, S. A. de C. V.; 2015. p. 71-151.

26. Puertas E, Rivera T. Factors that influence career choice in primary care among medical students starting social service in Honduras. Rev Panam Salud Publica. 2016;40(5):309-17.

27. Puertas E, Rodríguez Y, Alvarado E, et al. Career choice in primary care: Pre- and post-comparison of Honduran physicians completing social service. Rev Panam Salud Publica. 2017;41:e146

28. Royston PJ, Mathieson K, Leafman J, Ojan-Sheehan O. Medical student characteristics predictive of intent for rural practice. Rural Remote Health. 2012;12:2107.

29. Rabinowitz HK, Diamond JJ, Markham FW, Santana AJ. The relationship between entering medical students' backgrounds and career plans and their rural practice outcomes three decades later. Acad Med. 2012;87(4):493-7. doi: 10.1097/ACM.0b013e3182488c06

30. Scott I, Gowans M, Wright B, Brenneis F, Banner S, Boone J. Determinants of choosing a career in family medicine. Can Med Assoc J. 2011;183(1):E1-8.

31. Gill H, McLeod S, Duerksen K, Szafran O. Factors influencing medical students' choice of family medicine: Effects of rural versus urban background. Can Fam Physician. 2012;58(11):e649-57.

32. Kiolbassa K, Miksch A, Hermann K, et al. Becoming a general practitioner: Which factors have most impact on career choice of medical students? BMC Fam Pract. 2011;12:25-2296-12-25. doi: 10.1186/1471-2296-12-25

33. Feldman K, Woloschuk W, Gowans M, et al. The difference between medical students interested in rural family medicine versus urban family or specialty medicine. Can J Rural Med. 2008;13(2):73-9.

34. Wright B, Scott I, Woloschuk W, Brenneis F, Bradley J. Career choice of new medical students at three Canadian universities: Family medicine versus specialty medicine. CMAJ. 2004;170(13):1920-24.

35. Gibis B, Heinz A, Jacob R, Muller CH. The career expectations of medical students: Findings of a nationwide survey in Germany. Dtsch Arztebl Int. 2012;109(18):327-32. doi: 10.3238/arztebl.2012.0327

36. Lefevre JH. Career choices of medical students: A national survey of 1780 students. Med Educ. 2010;44(6):603-12.

37. Jeffe DB, Whelan AJ, Andriole DA. Primary care specialty choices of United States medical graduates, 1997-2006. Acad Med. 2010;85(6):947-58. doi: 10.1097/ACM.0b013e3181dbe77d

38. Puddester DG. Staying human in the medical family: The unique role of doctor-parents. Med J Aust. 2004;181(7):395-8.

39. Sarma S, Thind A, Chu MK. Do new cohorts of family physicians work less compared to their older predecessors? The evidence from Canada. Social Science \& Medicine (1982). 2011;72(12):2049-58. doi: 10.1016/j.socscimed.2011.03.047

40. Bennett KL, Phillips JP. Finding, recruiting, and sustaining the future primary care physician workforce: A new theoretical model of specialty choice process. Acad Med. 2010;85(10 Suppl):S81-8. doi: 10.1097/ACM.0b013e3181ed4bae

Manuscrito recibido el 8 de enero de 2020. Aceptado para su publicación, tras revisión, el 24 de junio de 2020. 


\section{Factors influencing the choice of a career in primary care among medical students in Central America}

ABSTRACT Objectives. To identify the preferred specialties, salary perception and other factors that influence the choice of a career in primary care among last-year medical students or social service students in Central America.

Methods. A cross-sectional, multicenter study using a survey that investigated demographic information, preferred specialty, salary perception, and other factors that influence the choice of a specialty.

Results. A total of 1722 students from 31 universities participated and identified as future specialties: surgery (11.7\%), gynecology/obstetrics (10.3\%), pediatrics (9.9\%) and internal medicine (6.6\%). General medicine was preferred by $3.8 \%$ and family medicine by $1.1 \%$. On grouping them, the greatest interest was observed for medical specialties (49.7\%), followed by surgical specialties (31.7\%). Primary care registered an interest of $17.1 \%$. Surgical specialties were perceived as having the highest income (USD 36 000); primary care was perceived as having the lowest income (USD 24 000). Income (23.6\%), future work (19.7\%) and "making a difference in people" (8.9\%) were the main factors involved in the choice. "Working with people with low access" was significantly associated with preference for primary care. Preference for other specialties was influenced by "perceived prestige" and "enjoying life" $(P<0.05)$. Most participants who chose primary care were studying at a public university $(P<0.05)$, highlighting the role of public institutions of higher education.

Conclusions. There is a combination of facilitating factors and barriers that affect the low interest in primary care careers. There is a need for strategies from academia and the government sector, as well as the definition of public policies, that support the choice of primary care.

Keywords $\quad$ Primary health care; specialization; health workforce; Central America. 